\title{
Stratified-algebraic vector bundles of small rank
}

\author{
WOJCIECH KUCHARZ
}

\begin{abstract}
We investigate vector bundles on real algebraic varieties. Our goal is to construct rank 2 real and complex stratified-algebraic vector bundles with prescribed Stiefel-Whitney and Chern classes, respectively. We obtain a partial solution of this problem and present two applications.
\end{abstract}

Mathematics Subject Classification. 14P25, 14P99, 14F25, 57R22.

Keywords. Real algebraic variety, Stratified-algebraic vector bundle, Stiefel-Whitney classes, Chern classes.

1. Introduction. In the recent joint paper with Kurdyka [14], we introduced and investigated stratified-algebraic vector bundles on real algebraic varieties. They occupy an intermediate position between algebraic and topological vector bundles. A hard problem is to find a characterization of topological vector bundles admitting a stratified-algebraic structure, cf. $[12,15]$. In the present paper we study rank 2 real and complex stratified-algebraic vector bundles with prescribed Stiefel-Whitney and Chern classes, respectively. As an application, we obtain a criterion for a topological complex vector bundle of rank 2 and a topological quaternionic line bundle to admit a stratified-algebraic structure. This paper fits into the new direction of research in real algebraic geometry developed by several authors [1,3,4,8-15].

We use the term real algebraic variety to mean a locally ringed space isomorphic to an algebraic subset of $\mathbb{R}^{n}$, for some $n$, endowed with the Zariski topology and the sheaf of real-valued regular functions (such an object is called an affine real algebraic variety in [2]). The class of real algebraic varieties is identical with the class of quasiprojective real algebraic varieties, cf. [2, Proposition 3.2.10, Theorem 3.4.4]. Morphisms of real algebraic varieties are called

W. Kucharz was partially supported by the National Science Centre, Poland, under Grant Number 2014/15/B/ST1/00046. He also acknowledges with gratitude support and hospitality of the Max-Planck-Institut für Mathematik in Bonn. 
regular maps. Each real algebraic variety carries also the Euclidean topology, which is induced by the usual metric on $\mathbb{R}$. Unless explicitly stated otherwise, all topological notions relating to real algebraic varieties refer to the Euclidean topology.

Let $X$ be a real algebraic variety. By a stratification of $X$ we mean a finite collection $\mathcal{S}$ of pairwise disjoint Zariski locally closed subvarieties whose union is $X$. Each subvariety in $\mathcal{S}$ is called a stratum.

Let $\mathbb{F}$ stand for $\mathbb{R}, \mathbb{C}$, or $\mathbb{H}$ (the quaternions). When convenient, $\mathbb{F}$ will be identified with $\mathbb{R}^{d(\mathbb{F})}$, where $d(\mathbb{F})=\operatorname{dim}_{\mathbb{R}} \mathbb{F}$.

For any nonnegative integer $n$, let $\varepsilon_{X}^{n}(\mathbb{F})$ denote the standard trivial $\mathbb{F}$ vector bundle on $X$ with total space $X \times \mathbb{F}^{n}$, where $X \times \mathbb{F}^{n}$ is regarded as a real algebraic variety.

An algebraic $\mathbb{F}$-vector bundle on $X$ is an algebraic $\mathbb{F}$-vector subbundle of $\varepsilon_{X}^{n}(\mathbb{F})$ for some $n$ (cf. [2, Chapters 12 and 13] for various characterizations of algebraic $\mathbb{F}$-vector bundles).

We now recall the fundamental notion introduced in [14]. A stratifiedalgebraic $\mathbb{F}$-vector bundle on $X$ is a topological $\mathbb{F}$-vector subbundle $\xi$ of $\varepsilon_{X}^{n}(\mathbb{F})$, for some $n$, such that for some stratification $\mathcal{S}$ of $X$, the restriction $\left.\xi\right|_{S}$ of $\xi$ to each stratum $S$ in $\mathcal{S}$ is an algebraic $\mathbb{F}$-vector subbundle of $\varepsilon_{S}^{n}(\mathbb{F})$.

A topological $\mathbb{F}$-vector bundle on $X$ is said to admit a stratified-algebraic structure if it is isomorphic to a stratified-algebraic $\mathbb{F}$-vector bundle on $X$.

As usual, we denote the $i$ th Stiefel-Whitney class of an $\mathbb{R}$-vector bundle $\eta$ by $w_{i}(\eta)$.

Problem 1.1. Given a positive integer $k$, characterize the cohomology classes $u_{i}$ in $H^{i}(X ; \mathbb{Z} / 2)$ for which there exists a rank $k$ stratified-algebraic $\mathbb{R}$-vector bundle $\xi$ on $X$ with $w_{i}(\xi)=u_{i}$ for $1 \leq i \leq k$.

For a $\mathbb{C}$-vector bundle $\eta$, let $c_{i}(\eta)$ denote its $i$ th Chern class.

Problem 1.2. Given a positive integer $k$, characterize the cohomology classes $u_{i}$ in $H^{2 i}(X ; \mathbb{Z})$ for which there exists a rank $k$ stratified-algebraic $\mathbb{C}$-vector bundle $\xi$ on $X$ with $c_{i}(\xi)=u_{i}$ for $1 \leq i \leq k$.

In [14] we solved Problems 1.1 and 1.2 for $k=1$. Presently, we address these problems for $k=2$ (Theorems 2.3 and 2.6). The case $k \geq 3$ seems to be out of reach. Our partial solution of Problem 1.2 leads to a criterion for a topological $\mathbb{C}$-vector bundle of rank 2 and a topological $\mathbb{H}$-line bundle to admit a stratified-algebraic structure (Theorems 2.7 and 2.8).

It would be interesting to obtain at least a partial solution of the counterparts of Problems 1.1 and 1.2 for $k=2$, where stratified-algebraic vector bundles are replaced by algebraic vector bundles. However, this would require some new approach, different from the methods used here.

2. Constructions of rank 2 vector bundles. For the convenience of the reader, we first review some classical topological constructions.

Convention Working with smooth (of class $\mathcal{C}^{\infty}$ ) manifolds, we always assume that submanifolds are closed subsets of the ambient manifold. 
Let $X$ be a smooth manifold, and let $M$ be a smooth codimension $k$ submanifold of $X$. Suppose that the normal bundle to $M$ in $X$ is oriented, and denote by $\tau_{M}^{X}$ the Thom class of $M$ in the cohomology group $H^{k}(X, X \backslash M ; \mathbb{Z})$, cf. [16, p. 118]. The image of $\tau_{M}^{X}$ by the restriction homomorphism $H^{k}(X, X \backslash$ $M ; \mathbb{Z}) \rightarrow H^{k}(X ; \mathbb{Z})$, induced by the inclusion map $X \hookrightarrow(X, X \backslash M)$, will be denoted by $\llbracket M \rrbracket^{X}$ and called the cohomology class represented by $M$. If $X$ is compact and oriented, and $M$ is endowed with the compatible orientation, then $\llbracket M \rrbracket^{X}$ is up to sign Poincaré dual to the homology class in $H_{*}(X ; \mathbb{Z})$ represented by $M$, cf. [16, p. 136]. Similarly, without any orientability assumption, we define the cohomology class $[M]^{X}$ in $H^{k}(X ; \mathbb{Z} / 2)$ represented by $M$. The cohomology class $[M]^{X}$ is Poincaré dual to the homology class in $H_{*}(X ; \mathbb{Z} / 2)$ represented by $M$.

Let $Y$ be a smooth manifold, and let $N$ be a smooth submanifold of $Y$. Let $f: X \rightarrow Y$ be a smooth map transverse to $N$. If the normal bundle to $N$ in $Y$ is oriented and the normal bundle to the smooth submanifold $M:=f^{-1}(N)$ of $X$ is endowed with the orientation induced by $f$, then $\tau_{M}^{X}=f^{*}\left(\tau_{N}^{Y}\right)$, where $f$ is regarded as a map from $(X, X \backslash M)$ into $(Y, Y \backslash N)$ (this follows from $[5$, p. 117, Theorem 6.7]). In particular,

$$
\llbracket M \rrbracket^{X}=f^{*}\left(\llbracket N \rrbracket^{Y}\right) .
$$

Without any orientability assumption,

$$
[M]^{X}=f^{*}\left([N]^{Y}\right) .
$$

Let $\xi$ be a rank $k$ smooth $\mathbb{R}$-vector bundle on $X$. A smooth section $s: X \rightarrow \xi$ is said to be transverse regular if it is transverse to the zero section of $\xi$. In that case, the zero locus of $s$,

$$
Z(s):=\{x \in X \mid s(x)=0\},
$$

is a smooth codimension $k$ submanifold of $\mathrm{X}$. We identify the normal bundle to $Z(s)$ in $X$ with $\left.\xi\right|_{Z(s)}$ via the isomorphism induced by $s$. In particular, if the vector bundle $\xi$ is oriented, then so is the normal bundle to $Z(s)$ in $X$ and

$$
e(\xi)=\llbracket Z(s) \rrbracket^{X},
$$

where $e(\xi)$ stands for the Euler class of $\xi$. Indeed, let $E$ be the total space of $\xi$ and $p: E \rightarrow X$ the bundle projection. Identify $X$ with the image of the zero section of $\xi$. The section $s$ is transverse to $X$ and $Z(s)=s^{-1}(X)$. Consequently, $\llbracket Z(s) \rrbracket^{X}=s^{*}\left(\llbracket X \rrbracket^{E}\right)$. Hence

$$
p^{*}\left(\llbracket Z(s) \rrbracket^{X}\right)=p^{*}\left(s^{*}\left(\llbracket X \rrbracket^{E}\right)\right)=(s \circ p)^{*}\left(\llbracket X \rrbracket^{E}\right)=\llbracket X \rrbracket^{E},
$$

where the last equality holds since $s \circ p: E \rightarrow E$ is homotopic to the identity map. On the other hand, $p^{*}(e(\xi))=\llbracket X \rrbracket^{E}$, cf. [16, p. 98]. It follows that $e(\xi)=\llbracket Z(s) \rrbracket^{X}$ since $p^{*}$ is an isomorphism. Similarly, if $\xi$ is not necessarily orientable, we get

$$
w_{k}(\xi)=[Z(s)]^{X} \text {. }
$$

Recall that on a smooth manifold each topological vector bundle is isomorphic to a smooth vector bundle, which is uniquely determined up to smooth isomorphism, cf. [5, p. 101]. 
For any rank $k \mathbb{F}$-vector bundle $\eta$, where $\mathbb{F}=\mathbb{R}$ or $\mathbb{F}=\mathbb{C}$, let $\operatorname{det} \eta$ denote the $k$ th exterior power of $\eta$. Thus $\operatorname{det} \eta$ is an $\mathbb{F}$-line bundle. Furthermore,

$$
\begin{aligned}
w_{1}(\operatorname{det} \eta)=w_{1}(\eta) & \text { if } \mathbb{F}=\mathbb{R}, \\
c_{1}(\operatorname{det} \eta)=c_{1}(\eta) & \text { if } \mathbb{F}=\mathbb{C},
\end{aligned}
$$

cf. [6, p. 64].

Given a subset $A$ of $X$ and a cohomology class $u$ in $H^{i}(X ; G)$, where $G=\mathbb{Z} / 2$ or $G=\mathbb{Z}$, we denote by $\left.u\right|_{A}$ the image of $u$ by the homomorphism $H^{i}(X ; G) \rightarrow H^{i}(A ; G)$ induced by the inclusion map $A \hookrightarrow X$.

Proposition 2.1. Let $X$ be a smooth manifold and let $\theta$ be a rank 2 topological $\mathbb{R}$-vector bundle on $X$. Then there exist smooth submanifolds $M_{i}$ of $X$ such that $\operatorname{codim}_{X} M_{i}=i$,

$$
w_{1}(\operatorname{det} \nu)=\left.\left[M_{1}\right]^{X}\right|_{M_{2}} \text { in } H^{1}\left(M_{2} ; \mathbb{Z} / 2\right),
$$

where $\nu$ is the normal bundle to $M_{2}$ in $X$, and

$$
w_{i}(\theta)=\left[M_{i}\right]^{X} \text { for } i=1,2 .
$$

Proof. We may assume without loss of generality that the vector bundle $\theta$ is smooth. Let

$$
s_{1}: X \rightarrow \operatorname{det} \theta, \quad s_{2}: X \rightarrow \theta
$$

be smooth transverse regular sections. Setting $M_{i}=Z\left(s_{i}\right)$, we get

$$
w_{1}(\theta)=w_{1}(\operatorname{det} \theta)=\left[M_{1}\right]^{X}, \quad w_{2}(\theta)=\left[M_{2}\right]^{X} .
$$

Furthermore, since we identify $\nu$ with $\left.\theta\right|_{M_{2}}$, we obtain $\operatorname{det} \nu=\left.(\operatorname{det} \theta)\right|_{M_{2}}$ and

$$
w_{1}(\operatorname{det} \nu)=w_{1}\left(\left.(\operatorname{det} \theta)\right|_{M_{2}}\right)=\left.\left[M_{1}\right]^{X}\right|_{M_{2}},
$$

as required.

Proposition 2.1 provides motivation for the following result.

Proposition 2.2. Let $X$ be a smooth manifold and let $M_{i}$ be a smooth submanifold of $X$ such that $\operatorname{codim}_{X} M_{i}=i$ for $i=1,2$, and

$$
w_{1}(\operatorname{det} \nu)=\left.\left[M_{1}\right]^{X}\right|_{M_{2}} \text { in } H^{1}\left(M_{2} ; \mathbb{Z} / 2\right),
$$

where $\nu$ is the normal bundle to $M_{2}$ in $X$. Then there exists a rank 2 smooth $\mathbb{R}$-vector bundle $\theta$ on $X$ with

$$
w_{i}(\theta)=\left[M_{i}\right]^{X} \text { for } i=1,2 .
$$

Furthermore, the vector bundle $\theta$ can be chosen so that there exist smooth transverse regular sections

$$
s_{1}: X \rightarrow \operatorname{det} \theta, \quad s_{2}: X \rightarrow \theta
$$

satisfying $Z\left(s_{i}\right)=M_{i}$ for $i=1,2$. 
Proof. Let $\lambda$ be a smooth $\mathbb{R}$-line bundle on $X$ with

$$
w_{1}(\lambda)=\left[M_{1}\right]^{X} .
$$

It is well known that there exists a smooth transverse regular section $u: X \rightarrow \lambda$ satisfying

$$
Z(u)=M_{1}
$$

Let $\rho: T \rightarrow M_{2}$ be a tubular neighborhood of $M_{2}$ in $X$. There exists a smooth transverse regular section $\sigma: T \rightarrow \rho^{*} \nu$ such that $Z(\sigma)=M_{2}$. In particular,

$$
\left.\left(\rho^{*} \nu\right)\right|_{T \backslash M_{2}}=\mu \oplus \varepsilon_{\sigma},
$$

where $\varepsilon_{\sigma}$ is the trivial $\mathbb{R}$-line subbundle of $\left.\left(\rho^{*} \nu\right)\right|_{T \backslash M_{2}}$ generated by $\left.\sigma\right|_{T \backslash M_{2}}$, and $\mu$ is a smooth $\mathbb{R}$-line bundle on $T \backslash M_{2}$. have

We assert that the $\mathbb{R}$-line bundles $\mu$ and $\left.\lambda\right|_{T \backslash M_{2}}$ are isomorphic. Indeed, we

$$
w_{1}(\nu)=w_{1}(\operatorname{det} \nu)=\left.\left[M_{1}\right]^{X}\right|_{M_{2}}=\left.w_{1}(\lambda)\right|_{M_{2}} .
$$

Consequently,

$$
w_{1}\left(\rho^{*} \nu\right)=w_{1}\left(\left.\lambda\right|_{T}\right)
$$

the map $\rho: T \rightarrow M_{2}$ being a homotopy inverse of the inclusion map $M_{2} \hookrightarrow T$. Hence

$$
w_{1}\left(\left.\left(\rho^{*} \nu\right)\right|_{T \backslash M_{2}}\right)=w_{1}\left(\left.\lambda\right|_{T \backslash M_{2}}\right) .
$$

On the other hand, in view of (3),

$$
w_{1}(\mu)=w_{1}\left(\left.\left(\rho^{*} \nu\right)\right|_{T \backslash M_{2}}\right) .
$$

By combining (4) and (5), we get

$$
w_{1}(\mu)=w_{1}\left(\left.\lambda\right|_{T \backslash M_{2}}\right),
$$

which implies the assertion, cf. [7, p. 234].

Let $\varepsilon$ be the standard trivial $\mathbb{R}$-line bundle on $X$ with total space $X \times \mathbb{R}$ and let $\tau: X \rightarrow \lambda \oplus \varepsilon$ be the smooth section defined by $\tau(x)=(0,(x, 1))$ for all $x$ in $X$. By the assertion, there exists a smooth isomorphism

$$
\varphi:\left.\left.\left(\rho^{*} \nu\right)\right|_{T \backslash M_{2}} \rightarrow(\lambda \oplus \varepsilon)\right|_{T \backslash M_{2}}
$$

such that $\varphi \circ \sigma=\tau$ on $T \backslash M_{2}$.

Let $\theta$ be the smooth $\mathbb{R}$-vector bundle on $X$ obtained by gluing $\rho^{*} \nu$ and $\left.(\lambda \oplus \varepsilon)\right|_{X \backslash M_{2}}$ over $T \backslash M_{2}$ using $\varphi$. Similarly, let $s_{2}: X \rightarrow \theta$ be the smooth section obtained by gluing $\sigma$ and $\left.\tau\right|_{X \backslash M_{2}}$ over $T \backslash M_{2}$ using $\varphi$. By construction, $\theta$ is a rank 2 smooth $\mathbb{R}$-vector bundle on $X$, the section $s_{2}$ is transverse regular, and

$$
Z\left(s_{2}\right)=M_{2} .
$$

Consequently,

$$
w_{2}(\theta)=\left[M_{2}\right]^{X} .
$$

In view of $(1),(2),(6),(7)$, it remains to prove that the $\mathbb{R}$-line bundles $\operatorname{det} \theta$ and $\lambda$ are isomorphic. To this end it suffices to show the equality

$$
w_{1}(\operatorname{det} \theta)=w_{1}(\lambda),
$$


cf. [7, p. 234]. Note that

$$
w_{1}\left(\left.(\operatorname{det} \theta)\right|_{X \backslash M_{2}}\right)=w_{1}\left(\left.\theta\right|_{X \backslash M_{2}}\right)=w_{1}\left(\left.(\lambda \oplus \varepsilon)\right|_{X \backslash M_{2}}\right)=w_{1}\left(\left.\lambda\right|_{X \backslash M_{2}}\right)
$$

and hence

$$
\left.w_{1}(\operatorname{det} \theta)\right|_{X \backslash M_{2}}=\left.w_{1}(\lambda)\right|_{X \backslash M_{2}} .
$$

As a portion of the long exact cohomology sequence of the pair $\left(X, X \backslash M_{2}\right)$, we get

$$
H^{1}\left(X, X \backslash M_{2} ; \mathbb{Z} / 2\right) \rightarrow H^{1}(X ; \mathbb{Z} / 2) \stackrel{e^{*}}{\longrightarrow} H^{1}\left(X \backslash M_{2} ; \mathbb{Z} / 2\right),
$$

where $e: X \backslash M_{2} \hookrightarrow X$ is the inclusion map. Since $H^{1}\left(X, X \backslash M_{2} ; \mathbb{Z} / 2\right)=0$ (cf. [16, pp. 106, 117], it follows that $e^{*}$ is a monomorphism. Hence (8) holds, as required.

If $X$ is a smooth manifold, by combining Propositions 2.1 and 2.2, we obtain a characterization of the cohomology classes $u_{i}$ in $H^{i}(X ; \mathbb{Z} / 2)$ for which there exists a rank 2 topological $\mathbb{R}$-vector bundle $\theta$ on $X$ with $w_{i}(\theta)=u_{i}$ for $i=1,2$. Our partial solution of Problem 1.1 is of a similar nature. We first recall a well-known phenomenon specific to real algebraic geometry. Namely, if $X$ is a nonsingular real algebraic variety, it can happen that a nonsingular Zariski locally closed subvariety of $X$ is Euclidean closed but not Zariski closed.

Theorem 2.3. Let $X$ be a compact nonsingular real algebraic variety, and let $M_{i}$ be a smooth submanifold of $X$ such that $\operatorname{codim}_{X} M_{i}=i$ for $i=1,2$, and

$$
w_{1}(\operatorname{det} \nu)=\left.\left[M_{1}\right]^{X}\right|_{M_{2}} \text { in } H^{1}\left(M_{2} ; \mathbb{Z} / 2\right),
$$

where $\nu$ is the normal bundle to $M_{2}$ in $X$. Assume that $M_{i}$ is a nonsingular Zariski locally closed subvariety of $X$ for $i=1,2$. Then there exists a rank 2 stratified-algebraic $\mathbb{R}$-vector bundle $\xi$ on $X$ with

$$
w_{i}(\xi)=[M]_{i}^{X} \quad \text { for } i=1,2 .
$$

Proof. It suffices to make use of Proposition 2.2 and [14, Theorem 1.9].

Let $\mathbb{K}$ be a subfield of $\mathbb{F}$, where $\mathbb{K}$ (as $\mathbb{F}$ ) stands for $\mathbb{R}$, $\mathbb{C}$, or $\mathbb{H}$. Any $\mathbb{F}$-vector bundle $\eta$ can be regarded as a $\mathbb{K}$-vector bundle, which is indicated by $\eta_{\mathbb{K}}$. In particular, $\eta_{\mathbb{K}}=\eta$ if $\mathbb{K}=\mathbb{F}$.

Suppose now that $\xi$ is a rank $k$ smooth $\mathbb{C}$-vector bundle on a smooth manifold $X$. Recall that

$$
c_{k}(\xi)=e\left(\xi_{\mathbb{R}}\right),
$$

where $\xi_{\mathbb{R}}$ is endowed with the orientation induced by the complex structure, cf. [16, p. 158]. If $s: X \rightarrow \xi$ is a smooth transverse regular section, then we regard the normal bundle to $Z(s)$ in $X$ as a $\mathbb{C}$-vector bundle, identifying it with $\left.\xi\right|_{Z(s)}$. In particular, the normal bundle to $Z(s)$ in $X$ is canonically oriented, the cohomology class $\llbracket Z(s) \rrbracket^{X}$ in $H^{2 k}(X ; \mathbb{Z})$ is defined, and

$$
c_{k}(\xi)=\llbracket Z(s) \rrbracket^{X} .
$$

If $M$ is a smooth codimension $2 k$ submanifold of $X$ and the normal bundle $\nu$ to $M$ in $X$ is endowed with a complex structure, then the $\mathbb{R}$-vector bundle 
$\nu_{\mathbb{R}}$ is canonically oriented and the cohomology class $\llbracket M \rrbracket^{X}$ in $H^{2 k}(X ; \mathbb{Z})$ is defined.

For $\mathbb{C}$-vector bundles, Proposition 2.1 takes the following form.

Proposition 2.4. Let $X$ be a smooth manifold, and let $\theta$ be a rank 2 topological $\mathbb{C}$-vector bundle on $X$. Then there exist smooth submanifolds $M_{i}$ of $X$ such that $\operatorname{codim}_{X} M_{i}=2 i$, the normal bundle $\nu_{i}$ to $M_{i}$ in $X$ is endowed with a complex structure,

$$
c_{1}\left(\operatorname{det} \nu_{2}\right)=\left.\llbracket M_{1} \rrbracket^{X}\right|_{M_{2}} \quad \text { in } H^{2}\left(M_{2} ; \mathbb{Z}\right),
$$

and $c_{i}(\theta)=\llbracket M_{i} \rrbracket^{X}$ for $i=1,2$.

Proof. We may assume without loss of generality that the vector bundle $\theta$ is smooth. Let

$$
s_{1}: X \rightarrow \operatorname{det} \theta, \quad s_{2}: X \rightarrow \theta
$$

be smooth transverse regular sections. Setting $M_{i}=Z\left(s_{i}\right)$, we get

$$
c_{1}(\theta)=c_{1}(\operatorname{det} \theta)=\llbracket M_{1} \rrbracket^{X}, \quad c_{2}(\theta)=\llbracket M_{2} \rrbracket^{X} .
$$

Furthermore, since we identify $\nu_{2}$ with $\left.\theta\right|_{M_{2}}$, we obtain $\operatorname{det} \nu_{2}=\left.(\operatorname{det} \theta)\right|_{M_{2}}$ and

$$
c_{1}\left(\operatorname{det} \nu_{2}\right)=c_{1}\left(\left.(\operatorname{det} \theta)\right|_{M_{2}}\right)=\left.\llbracket M_{1} \rrbracket^{X}\right|_{M_{2}},
$$

as required.

The following is a counterpart of Proposition 2.2 for $\mathbb{C}$-vector bundles.

Proposition 2.5. Let $X$ be a smooth manifold, and let $M_{i}$ be a smooth submanifold of $X$ such that $\operatorname{codim}_{X} M_{i}=2 i$, the normal bundle $\nu_{i}$ to $M_{i}$ in $X$ is endowed with a complex structure for $i=1,2$, and

$$
c_{1}\left(\operatorname{det} \nu_{2}\right)=\left.\llbracket M_{1} \rrbracket^{X}\right|_{M_{2}} \text { in } H^{2}\left(M_{2} ; \mathbb{Z}\right) .
$$

Then there exists a rank 2 smooth $\mathbb{C}$-vector bundle $\theta$ on $X$ with

$$
c_{i}(\theta)=\llbracket M_{i} \rrbracket^{X} \text { for } i=1,2 .
$$

Furthermore, the vector bundle $\theta$ can be chosen so that there exist smooth transverse regular sections

$$
s_{1}: X \rightarrow \operatorname{det} \theta, \quad s_{2}: X \rightarrow \theta
$$

satisfying $Z\left(s_{i}\right)=M_{i}$ for $i=1,2$.

Proof. The argument is analogous to that in the proof of Proposition 2.2. Let $\lambda$ be a smooth $\mathbb{C}$-line bundle on $X$ with

$$
c_{1}(\lambda)=\llbracket M_{1} \rrbracket^{X} .
$$

By $[14$, Lemma 8.20], there exists a smooth transverse regular section $u: X \rightarrow$ $\lambda$ satisfying

$$
Z(u)=M_{1} \text {. }
$$

Let $\rho: T \rightarrow M_{2}$ be a tubular neighborhood of $M_{2}$ in $X$. There exists a smooth transverse regular section $\sigma: T \rightarrow \rho^{*} \nu_{2}$ such that $Z(\sigma)=M_{2}$. In particular,

$$
\left.\left(\rho^{*} \nu_{2}\right)\right|_{T \backslash M_{2}}=\mu \oplus \varepsilon_{\sigma},
$$


where $\varepsilon_{\sigma}$ is the trivial $\mathbb{C}$-line subbundle of $\left.\left(\rho^{*} \nu_{2}\right)\right|_{T \backslash M_{2}}$ generated by $\left.\sigma\right|_{T \backslash M_{2}}$, and $\mu$ is a smooth $\mathbb{C}$-line bundle on $T \backslash M_{2}$.

We assert that the $\mathbb{C}$-line bundles $\mu$ and $\lambda_{T \backslash M_{2}}$ are isomorphic. Indeed, we have

$$
c_{1}\left(\nu_{2}\right)=c_{1}\left(\operatorname{det} \nu_{2}\right)=\left.\llbracket M_{1} \rrbracket^{X}\right|_{M_{2}}=\left.c_{1}(\lambda)\right|_{M_{2}} .
$$

Consequently,

$$
c_{1}\left(\rho^{*} \nu_{2}\right)=c_{1}\left(\left.\lambda\right|_{T}\right)
$$

the map $\rho: T \rightarrow M_{2}$ being a homotopy inverse of the inclusion map $M_{2} \hookrightarrow T$. Hence

$$
c_{1}\left(\left.\left(\rho^{*} \nu_{2}\right)\right|_{T \backslash M_{2}}\right)=c_{1}\left(\left.\lambda\right|_{T \backslash M_{2}}\right) .
$$

On the other hand, in view of (3),

$$
c_{1}(\mu)=c_{1}\left(\left.\left(\rho^{*} \nu_{2}\right)\right|_{T \backslash M_{2}}\right) .
$$

By combining (4) and (5), we get

$$
c_{1}(\mu)=c_{1}\left(\left.\lambda\right|_{T \backslash M_{2}}\right),
$$

which implies the assertion, cf. [7, p. 234].

Let $\varepsilon$ be the standard trivial $\mathbb{C}$-line bundle on $X$ with total space $X \times \mathbb{C}$, and let $\tau: X \rightarrow \lambda \oplus \varepsilon$ be the smooth section defined by $\tau(x)=(0,(x, 1))$ for all $x$ in $X$. By the assertion, there exists a smooth isomorphism

$$
\varphi:\left.\left.\left(\rho^{*} \nu_{2}\right)\right|_{T \backslash M_{2}} \rightarrow(\lambda \oplus \varepsilon)\right|_{T \backslash M_{2}}
$$

such that $\varphi \circ \sigma=\tau$ on $T \backslash M_{2}$.

Let $\theta$ be the smooth $\mathbb{C}$-vector bundle on $X$ obtained by gluing $\rho^{*} \nu_{2}$ and $\left.(\lambda \oplus \varepsilon)\right|_{X \backslash M_{2}}$ over $T \backslash M_{2}$ using $\varphi$. Similarly, let $s_{2}: X \rightarrow \theta$ be the smooth section obtained by gluing $\sigma$ and $\left.\tau\right|_{X \backslash M_{2}}$ over $T \backslash M_{2}$ using $\varphi$. By construction, $\theta$ is a rank 2 smooth $\mathbb{C}$-vector bundle on $X$, the section $s_{2}$ is transverse regular, and

$$
Z\left(s_{2}\right)=M_{2}
$$

Consequently,

$$
c_{2}(\theta)=\llbracket M_{2} \rrbracket^{X} .
$$

In view of $(1),(2),(6),(7)$, it remains to prove that the $\mathbb{C}$-line bundles $\operatorname{det} \theta$ and $\lambda$ are isomorphic. To this end, it suffices to show the equality

$$
c_{1}(\operatorname{det} \theta)=c_{1}(\lambda)
$$

cf. [7, p. 234]. Note that

$$
c_{1}\left(\left.(\operatorname{det} \theta)\right|_{X \backslash M_{2}}\right)=c_{1}\left(\left.\theta\right|_{X \backslash M_{2}}\right)=c_{1}\left(\left.(\lambda \oplus \varepsilon)\right|_{X \backslash M_{2}}\right)=c_{1}\left(\left.\lambda\right|_{X \backslash M_{2}}\right)
$$

and hence

$$
\left.c_{1}(\operatorname{det} \theta)\right|_{X \backslash M_{2}}=\left.c_{1}(\lambda)\right|_{X \backslash M_{2}} .
$$

As a portion of the long exact cohomology sequence of the pair $\left(X, X \backslash M_{2}\right)$, we get

$$
H^{2}\left(X, X \backslash M_{2} ; \mathbb{Z}\right) \rightarrow H^{2}(X ; \mathbb{Z}) \stackrel{e^{*}}{\longrightarrow} H^{2}\left(X \backslash M_{2} ; \mathbb{Z}\right),
$$


where $e: X \backslash M_{2} \hookrightarrow X$ is the inclusion map. Since $H^{2}\left(X, X \backslash M_{2} ; \mathbb{Z}\right)=0$ (cf. $\left[16\right.$, pp. 110, 117]), it follows that $e^{*}$ is a monomorphism. Hence (8) holds, as required.

If $X$ is a smooth manifold, then Propositions 2.4 and 2.5 yield a characterization of the cohomology classes $u_{i}$ on $H^{2 i}(X ; \mathbb{Z})$ for which there exists a rank 2 topological $\mathbb{C}$-vector bundle $\theta$ on $X$ with $c_{i}(\theta)=u_{i}$ for $i=1,2$.

Our partial solution of Problem 1.2 is the following.

Theorem 2.6. Let $X$ be a compact nonsingular real algebraic variety, and let $M_{i}$ be a smooth submanifold of $X$ such that $\operatorname{codim}_{X} M_{i}=2 i$, the normal bundle $\nu_{i}$ to $M_{i}$ in $X$ is endowed with a complex structure for $i=1,2$, and

$$
c_{1}\left(\operatorname{det} \nu_{2}\right)=\left.\llbracket M_{1} \rrbracket^{X}\right|_{M_{2}} \text { in } H^{2}\left(M_{2} ; \mathbb{Z}\right) .
$$

Assume that $M_{i}$ is a nonsingular Zariski locally closed subvariety of $X$ for $i=1,2$. Then there exists a rank 2 stratified-algebraic $\mathbb{C}$-vector bundle $\xi$ on $X$ with

$$
c_{i}(\xi)=\llbracket M_{i} \rrbracket^{X} \text { for } i=1,2 .
$$

Proof. It suffices to make use of Proposition 2.5 and [14, Theorem 1.9].

We conclude this paper by giving two applications of Theorem 2.6.

Theorem 2.7. Let $X$ be a compact nonsingular real algebraic variety, and let $\theta$ be a rank 2 topological $\mathbb{C}$-vector bundle on $X$. Let $M_{i}$ be smooth submanifolds of $X$ such that $\operatorname{codim}_{X} M_{i}=2 i$, the normal bundle $\nu_{i}$ to $M_{i}$ in $X$ is endowed with a complex structure,

$$
c_{1}\left(\operatorname{det} \nu_{2}\right)=\left.\llbracket M_{1} \rrbracket^{X}\right|_{M_{2}} \text { in } H^{2}\left(M_{2} ; \mathbb{Z}\right),
$$

and $c_{i}(\theta)=\llbracket M_{i} \rrbracket^{X}$ for $i=1,2$. Assume that $M_{i}$ is a nonsingular Zariski locally closed subvariety of $X$ for $i=1,2$. If for each integer $k \geq 3$ the only torsion in the cohomology group $H^{2 k}(X ; \mathbb{Z})$ is relatively prime to $(k-1)$ !, then $\theta$ admits a stratified-algebraic structure.

Proof. According to Theorem 2.6, there exists a rank 2 stratified-algebraic $\mathbb{C}$-vector bundle $\xi$ on $X$ with $c_{i}(\xi)=c_{i}(\theta)$ for $i=1,2$. Consequently,

$$
c_{k}(\xi)=c_{k}(\theta) \text { for all } k \geq 0 .
$$

Hence, if the condition on the torsion in the cohomology groups $H^{2 k}(X ; \mathbb{Z})$ is satisfied, then the $\mathbb{C}$-vector bundles $\xi$ and $\theta$ are stably equivalent, cf. [17, Theorem 3.2]. This implies, in view of [14, Corollary 3.14], that $\theta$ admits a stratified-algebraic structure.

The second application concerns $\mathbb{H}$-line bundles.

Theorem 2.8. Let $X$ be a compact nonsingular real algebraic variety, and let $\lambda$ be a topological $\mathbb{H}$-line bundle on $X$. Let $M$ be a smooth submanifold of $X$ such that $\operatorname{codim}_{X} M=4$, the normal bundle $\nu$ to $M$ in $X$ is endowed with a complex structure, $c_{1}(\operatorname{det} \nu)=0$ in $H^{2}(M ; \mathbb{Z})$, and $c_{2}\left(\lambda_{\mathbb{C}}\right)=\llbracket M \rrbracket^{X}$. Assume that $M$ is a nonsingular Zariski locally closed subvariety of $X$. If for each 
integer $k \geq 3$ the only torsion in the cohomology group $H^{2 k}(X ; \mathbb{Z})$ is relatively prime to $(k-1)$ !, then $\lambda$ admits a stratified-algebraic structure.

Proof. Suppose that the condition on the torsion in the cohomology groups $H^{2 k}(X ; \mathbb{Z})$ is satisfied. By Theorem 2.7, the $\mathbb{C}$-vector bundle $\lambda_{\mathbb{C}}$ admits a stratified-algebraic structure. Hence, in view of [14, Theorem 1.7], the $\mathbb{H}$-line bundle $\lambda$ admits a stratified-algebraic structure.

Open Access. This article is distributed under the terms of the Creative Commons Attribution 4.0 International License (http://creativecommons.org/licenses/ by/4.0/), which permits unrestricted use, distribution, and reproduction in any medium, provided you give appropriate credit to the original author(s) and the source, provide a link to the Creative Commons license, and indicate if changes were made.

\section{References}

[1] M. Bilski, W. Kucharz, A. Valette, and G. Valette, Vector bundles and regulous maps, Math. Z. 275 (2013), 403-418.

[2] J. Bochnak, M. Coste, And M.-F. Roy, Real Algebraic Geometry, Ergeb. der Math. und ihrer Grenzgeb. Folge 3, vol. 36, Springer, Berlin, 1998.

[3] G. Fichou, J. Huisman, F. Mangolte, and J.-Ph. Monnier, Fonctions régulues, arXiv:1112.3800 [math.AG], to appear in J. Reine Angew. Math.

[4] G. Fichou, J.-Ph. Monnier, and R. Quarez, Continuous functions in the plane regular after one blowing-up, arXiv:1409.8223 [math.AG].

[5] M. Hirsch, Differential Topology, Springer, New York, 1976.

[6] F. Hirzebruch, Topological Methods in Algebraic Geometry, Springer, Berlin, 1978.

[7] D. Husemoller, Fibre Bundles, Springer, New York-Heidelberg, 1975.

[8] J. Kollár ANd K. NowaK, Continuous rational functions on real and $p$-adic varieties, Math. Z. 279 (2015), 85-97.

[9] W. Kucharz, Rational maps in real algebraic geometry, Adv. Geom. 9 (2009), $517-539$.

[10] W. Kucharz, Approximation by continuous rational maps into spheres, J. Eur. Math. Soc. 16 (2014), 1555-1569.

[11] W. Kucharz, Continuous rational maps into the unit 2-sphere, Arch. Math. 102 (2014), 257-261.

[12] W. Kucharz, Some conjectures on stratified-algebraic vector bundles, J. Singul. 12 (2015), 92-104.

[13] W. Kucharz, Continuous rational maps into spheres, arXiv:1403.5127 [math.AG].

[14] W. Kucharz AND K. KurdyKa, Stratified-algebraic vector bundles, arXiv:1308.4376 [math.AG], to appear in J. Reine Angew. Math.

[15] W. Kucharz and K. Kurdyka, Comparison of stratified-algebraic and topological K-theory, arXiv:1511.04238 [math.AG]. 
[16] J.W. Milnor and J.D. Stasheff, Characteristic Classes, Princeton University Press, Princeton, NJ, 1974.

[17] F.P. Peterson, Some remarks on Chern classes, Ann. of Math. 69 (1959), 414-420.

\section{WOJCIECH KUCHARZ}

Institute of Mathematics Faculty of Mathematics and Computer Science, Jagiellonian University,

Łojasiewicza 6,

30-348 Kraków,

Poland

e-mail: Wojciech.Kucharz@im.uj.edu.pl

Received: 18 November 2015 